\title{
Sparmaßnahmen trotz Rekordüberschüssen? Zur Finanzierung der Arbeitsmarktpolitik
}

\author{
Judith Aust \\ Till Müller-Schoell
}

\begin{abstract}
Die Bundesagentur für Arbeit (BA) schloss das Haushaltsjahr 2006 mit einem Rekordüberschuss von 11,2 Milliarden $€$ ab. Dieses Finanzpolster dient jetzt als Anschubfinanzierung für die Beitragssatzsenkung 2007. Gleichzeitig wurde im Haushaltsbegleitgesetz die Abschaffung der Defizithaftung des Bundes gegenüber der BA vereinbart. Diese Beschlüsse sind für eine koordinierte Bewältigung der Arbeitsmarktprobleme nicht förderlich. Wer profitiert von einer zunehmenden Entsolidarisierung der Finanzierungsstrukturen? Wer trägt die Lasten der Sparpolitik? Kann Arbeitsmarktpolitik ihrem gesamtgesellschaftlichen Anspruch auf diese Weise noch gerecht werden?
\end{abstract}

\section{Einleitung}

Das Jahr 2006 war für die BA ein gutes Jahr: Überschüsse statt Defizite. Trotz dieser im Prinzip positiven Nachricht reißt die Kritik an der Finanzierung der Arbeitsmarktpolitik nicht ab. Einerseits klingt die Debatte um eine Kostenexplosion durch Hartz IV und Sozialmissbrauch noch nach. Andererseits sind Ursache und Verwendung des Überschusses strittig: Obwohl im Schnitt 4,5 Mio. Arbeitslose registriert wurden, blieben sowohl bei der BA als auch bei den für Hartz-IV Empfängern zuständigen Arbeitsgemeinschaften mehr als $15 \%$ der zur Verfügung stehenden Mittel für die Arbeitsförderung und Eingliederung von Arbeitslosen ungenutzt (Adamy 2007). Je stärker die eingesparten Mittel als Resultat einer Erholung am Arbeitsmarkt interpretiert werden, desto umstandsloser kann die Senkung von Sozialversicherungsbeiträgen gefordert werden. Mit Blick auf die Kürzungen bei der aktiven Arbeitsmarktpolitik und die einmaligen Zusatzeinnahmen durch die vorgezogene Beitragsleistung im Jahr 2006 sind Beitragssatzsenkungen jedoch kritisch zu diskutieren. Mehr finanzielles Engagement bei aktiven Maßnahmen scheint stattdessen angebracht.

Der Beitrag beleuchtet die Verschiebungen der Finanzierungsstrukturen in der Arbeitsmarktpolitik und bringt sie systematisch mit der Trennung in zwei Rechtskreise $^{1}$ und dem tendenziellen Rückzug des Bundes auf regulierende Aufgaben in Verbindung. Gezeigt wird, dass die erfolgte organisatorische Trennung in separate finanzielle Regelkreise in institutionellem Egois- mus mündet, der zulasten einer problemadäquaten Arbeitsmarktpolitik geht. In Abschnitt 2 beschreiben wir, ausgehend von einem kurzen Blick auf die Sozialleistungsquote, die Veränderung der Finanzierungsstrukturen zwischen einzelnen Sozialversicherungsträgern und damit verbundene Konsequenzen. Im Mittelpunkt des anschließenden Teils steht die Lastenverteilung zwischen bundesstaatlicher und kommunaler Ebene. Kommunen sind erheblich an den Kosten der Unterkunft im SGB II beteiligt. In Abschnitt 4 verengt sich der Fokus auf die zwei Regelkreise der Arbeitsmarktpolitik: Arbeitslosenversicherung und Grundsicherung von erwerbsfähigen Arbeitslosen werden auf Ausgabenentwicklung und Umfang des Leistungsbezugs hin untersucht. Im fünften Abschnitt wird der Rückzug des Bundes kritisch analysiert. Im sechsten Teil werden die fiskalischen Gewinner und Verlierer der letzten Jahre aufgeschlüsselt. Der Beitrag schließt mit einer zusammenfassenden Bewertung der Hartz-Reformen unter Finanzierungsaspekten (Abschnitt 7).

\section{Entsolidarisierte Finanzierungsstrukturen}

Auf gesamtstaatlicher Ebene zeigen die Sozialausgaben ebenso wie die von Arbeitnehmern und Arbeitgebern gezahlten Gesamtversicherungsbeiträge eine bemerkenswerte Stabilität. Die Sozialleistungsquote hat sich zwischen 2003 und 2005 nur leicht von $32,2 \%$ auf $31,0 \%$ reduziert. Auch die Gesamtversicherungsbeiträge liegen über das letzte Jahrzehnt hinweg be- trachtet ohne größere Schwankungen bei etwas mehr als $40 \%$.

Die Beitragssatzstabilität fußte auf einem lange Zeit funktionierenden sozialpolitischen Bündnis zwischen den verschiedenen Versicherungszweigen in der Sozialpolitik. Mit Blick auf den Gesamtbeitragssatz wurden die Beitragssätze einzelner Versicherungszweige so umgestaltet, dass Quersubventionen zwischen Versicherungen (und auch dem Bundeshaushalt) zum jeweils bedürftigen Zweig üblich waren (Trampusch 2003). Dieses Bündnis scheint sich gegenwärtig zugunsten sektoraler Interessenverfolgung aufzulösen, wie insbesondere anhand der Abschaffung der Defizithaftung gegenüber der BA oder der Diskussion um die Verwendung der BA-Überschüsse deutlich wird.

Die im Haushaltsbegleitgesetz geplante Abschaffung der Defizithaftung gegenüber der BA ist aus finanzierungstechnischen

\footnotetext{
1 Im SGB III sind die beitragsfinanzierten Leistungen des Arbeitslosengelds I inklusive Vermittlung, Beratung, Arbeitsförderung und Qualifizierung geregelt. Das SGB II umfasst die steuerfinanzierten Leistungen der Grundsicherung für arbeitsfähige Erwerbslose mit ALG II, Vermittlung und arbeitsmarktpolitische Maßnahmen.
}

Judith Aust, Dr., ist Wissenschaftlerin im WSI in der Hans-Böckler-Stiftung. Arbeitsschwerpunkte: Arbeitsmarktpolitik, vergleichende Wohlfahrtsstaatsforschung. e-mail: Judith-Aust@boeckler.de Till Müller-Schoell, Dr., ist Wissenschaftler im WSI in der Hans-Böckler-Stiftung. Arbeitsschwerpunkte: Arbeitsmarktpolitik, vergleichende Wohlfahrtsstaatsforschung. e-mail: Till-Mueller-Schoell@boeckler.de 
Tabelle 1: Salden der öffentlichen Haushalte 2004-2006

\begin{tabular}{|c|c|c|c|c|c|c|c|c|c|c|}
\hline & \multicolumn{2}{|c|}{ Staat } & \multicolumn{2}{|c|}{ Bund } & \multicolumn{2}{|c|}{ Länder } & \multicolumn{2}{|c|}{ Gemeinden } & \multicolumn{2}{|c|}{ Sozialversicherungen } \\
\hline & $\begin{array}{l}\text { Saldo } \\
\text { Mrd. } €\end{array}$ & $\begin{array}{c}\text { Veränderung } \\
(\%)\end{array}$ & $\begin{array}{l}\text { Saldo } \\
\text { Mrd. } €\end{array}$ & $\begin{array}{c}\text { Veränderung } \\
(\%)\end{array}$ & $\begin{array}{l}\text { Saldo } \\
\text { Mrd. } €\end{array}$ & $\begin{array}{c}\text { Veränderung } \\
(\%)\end{array}$ & $\begin{array}{l}\text { Saldo } \\
\text { Mrd. } €\end{array}$ & $\begin{array}{c}\text { Veränderung } \\
(\%)\end{array}$ & $\begin{array}{l}\text { Saldo } \\
\text { Mrd. } €\end{array}$ & $\begin{array}{c}\text { Veränderung } \\
(\%)\end{array}$ \\
\hline 2004 & $-82,46$ & & $-52,20$ & & $-26,78$ & & $-2,10$ & & $-1,38$ & \\
\hline 2005 & $-72,38$ & 10,08 & $-47,50$ & 4,70 & $-20,45$ & 6,33 & $-0,99$ & 1,11 & $-3,44$ & $-2,06$ \\
\hline 2006 & $-46,49$ & 25,89 & $-37,17$ & 10,33 & $-12,53$ & 7,92 & $-0,65$ & 0,34 & 3,86 & 7,30 \\
\hline
\end{tabular}

Gründen problematisch. Die BA kann nun auch in finanziell schwierigen Zeiten keine Bundeszuschüsse mehr zum Ausgleich ihres Haushalts erhalten. Finanzielle Engpässe infolge konjunktureller Risiken, unerwartete Beitragseinbrüche oder nicht vorhersehbare Ausgabensprünge bei gesetzlichen Pflichtleistungen werden nun isoliert zu Problemen der Arbeitslosenversicherung erklärt - und dies obwohl sie nach wie vor mit versicherungsfremden Ausgaben belastet ist und die unmittelbare Einflussnahme des Bundes auf die Geschäftspolitik fortbesteht (Adamy 2007). Vor diesem Hintergrund bleibt fraglich, ob die Arbeitslosenversicherung in der Lage ist, das finanzielle Risiko selbstständig zu tragen.

Während die Arbeitslosenversicherung in den letzten zwei Jahrzehnten stets Defizite machte, soll diese Phase jetzt durch einen Nettotransfer der Beitragszahler hin zum Steuerstaat beendet werden. Der Bundeszuschuss hatte 1992 einen Spitzenwert von $12,5 \mathrm{Mrd}$. $€$ erreicht. Im Jahr 2003 betrug er immerhin noch 6,2 Mrd. €, d.h. mindestens $10 \%$ des Haushalts der BA wurden aus dem Bundeshaushalt bestritten. Unter Berücksichtigung des Aussteuerungsbetrags - einer Leistung, die von der BA an den Bund gezahlt werden muss, wenn Arbeitlose vom Versicherungssystem ins Fürsorgesystem überwechseln - konnte der Bund im Jahr 2006 mehr als 10 Mrd. $€$ an Sozialbeiträgen aus der Arbeitslosenversicherung herausziehen. Selbst die Refinanzierung der letzten Beitragssenkung durch die Mehrwertsteuer bewirkte netto nur einen steuerfinanzierten Zuschuss zum Versicherungssystem von 1 bis $2 \mathrm{Mrd}$ €.

Im Geiste des tradierten Verschiebebahnhofs fordern die gesetzlichen Krankenkassen (GKV) einen Anteil an den Überschüssen der BA für ihre eigene Sanierung ein. Durch niedrige, nicht kostendeckende Beiträge für Arbeitslose - so das Argument - würden sie bereits seit langem die BA quersubventionieren (FAZ 29.8.2006, S.13). Das Ende des sozialpolitischen Bündnisses zeigt sich nun darin, dass trotz offenkundigem Finanzbedarfs bei den Krankenkassen der von der BA erwirtschaftete Überschuss nicht mehr zur Umgewichtung zugunsten der GKV genutzt wird. Vielmehr verwendet die BA den Überschuss einerseits zur hauseigenen Sanierung und gibt andererseits über Beitragssenkungen einen Teil des Überschusses an die Versichertengemeinschaft zurück. Dem Prinzip der sektoralen Interessenvertretung folgend ist davon auszugehen, dass mögliche Finanzierungsdefizite auf die Kunden der GKV verlagert werden und sich damit Versorgungsdefizite aufbauen. Verursacht wird der Finanzbedarf der GKV allerdings nur zu einem kleinen Teil von den Arbeitslosen. Bezieher des Arbeitslosengeldes I werden verbeitragt, d.h. Grundlage der Beitragsberechnung sind $80 \%$ des Entgelts, das zur Festsetzung der Arbeitslosenversicherungsleistung herangezogen wurde. ALG II-Empfänger werden steuerfinanziert in der GKV versichert und führen einen einheitlichen Beitrag von $120 €$ an die Krankenkassen ab. Auch wenn quantifizierbare Analysen derzeit nicht vorliegen, können wir davon ausgehen, dass insbesondere die Gruppe der Langzeitarbeitslosen mit hohen gesundheitlichen Risiken belastet ist und - entsprechend des gesellschaftspolitischen Auftrags der GKV - zu sehr günstigen Konditionen mitversichert wird. Dies ist jedoch soweit abschätzbar - bisher nahezu kostendeckend geschehen (Paquet/van Stiphout 2006, S. 193f.). Eine ähnliche Problematik stellt sich für die gesetzliche Rentenversicherung (GRV). Der Beitrag, der im Rahmen des ALG II-Bezugs an die Rentenversicherungsträger abgeführt werden muss, wurde 2007 von $78 €$ auf $40,80 €$ pro Monat gesenkt. Daraus folgen für die GRV geschätzte Beitragsausfälle in Höhe von ca. 2 Mrd. € pro Jahr, was ungefähr 0,2 Beitragspunkten entspricht (Ruland 2006, S. 228). Nimmt man den Wegfall der Doppelversicherung von Aufstockern ${ }^{2}$ (als Erwerbstätige und als Sozialleistungsbezieher) hinzu, liegt der Einnahmeausfall der GRV noch höher.

\section{Sanierungspolitik zulasten der Kommunen?}

Die Finanzlage des Staates hat sich in den letzten Jahren verbessert: Das jährliche Defizit fiel 2006 mit $46 \mathrm{Mrd}$. $€$ um $36 \mathrm{Mrd}$. $€$ niedriger aus als 2004 (Tabelle 1). Dieser Konsolidierungskurs ist allerdings ungleich auf die einzelnen Haushalte verteilt: Die Sozialversicherungen konnten Überschüsse erwirtschaften. Bund und Länder machten - in ihren größeren Etats - zusammen fast 30 Mrd. weniger Schulden. Demgegenüber steht ein Abbau des kommunalen Defizits um lediglich 1,5 Mrd. $€$.

Eine Ursache dieser Entwicklung ist in der zunehmenden Bedeutung von Sozialleistungen im Aufgabenbereich der Kommunen zu sehen. Zwischen 1979 und 2004 stiegen die Ausgaben der westdeutschen Kommunen für Sozialleistungen um $245 \%$. Gleichzeitig verdoppelten sich die Steuereinnahmen (Künast/Andreae 2007). Im Jahr 1997 gaben westdeutsche Kommunen 14,5\% und ostdeutsche Kommunen 21,1\% ihres Budgets für Soziales aus (Naßmacher/ Naßmacher 1999, S. 257). Heute liegt der gesamtdeutsche Durchschnitt bei $23,9 \%$.

Die Umverteilung der Kosten für die Grundsicherung im SGB II im Zuge der Zusammenlegung von Arbeitslosenhilfe und Sozialhilfe verstärkte den Trend zu wachsenden Sozialausgaben der Kommunen (Tabelle 2).

Die Grundsicherung wird aus Bundesmitteln finanziert. Die Kosten der Unterkunft werden jedoch nur zu knapp einem Drittel vom Bund übernommen (29,1\%) und in Form von Zuweisungen an die Kommunen ausgezahlt. Die Kommunen (und die BA) verwalten diese Mittel. Die Kommunen selbst beteiligen sich mit zwei Dritteln an der Finanzierung dieser Leis-

\footnotetext{
2 Aufstocker: Bezieher von Niedrigeinkommen, die
} Anrecht auf ergänzende Sozialleistungen haben. 
Tabelle 2: Einnahmen und Ausgaben der Kommunen 2003 bis 2007

\begin{tabular}{|c|c|c|c|c|c|}
\hline & \multicolumn{2}{|c|}{ Einnahmen } & \multicolumn{3}{|c|}{ Ausgaben } \\
\hline & insgesamt $^{2)}$ & $\begin{array}{c}\text { laufende } \\
\text { Zuweisungen2) }\end{array}$ & insgesamt ${ }^{2)}$ & Soziales') & Anteil in \% \\
\hline 2003 & 141,4 & 38,0 & 149,8 & 30,4 & 20,3 \\
\hline 2004 & 145,4 & 38,8 & 149,2 & 31,9 & 21,2 \\
\hline 2005 & 151,1 & 42,7 & 153,4 & 35,5 & 23,1 \\
\hline 2006 & 158,0 & 43,1 & 156,3 & 37,4 & 23,9 \\
\hline 20071) & 160,8 & 45,6 & 159,5 & 38,5 & 24,1 \\
\hline
\end{tabular}

tung $(70,9 \%)$. Diese Finanzierungsstruktur führt aus zwei Gründen tendenziell zu einer Entlastung des Bundes und einer gleichzeitig stärkeren Belastung der Kommunen: Zum einen sind es die der Leistungsberechnung zugrunde gelegten Miethöhen, die in vielen Regionen unterhalb des tatsächlichen Marktpreises liegen (Wahrendorf 2006). Da Kosten der Unterkunft von den Kommunen voll zu übernehmen sind, müssen diese den finanziellen Mehraufwand, der sich aus dem höheren kommunalen Mietspiegel ergibt, voll ausgleichen. Ein zweiter Grund ist in der wachsenden Zahl der Aufstocker zu sehen. Das zu berücksichtigende Einkommen und Vermögen der Leistungsbezieher mindert nämlich zunächst die über Bundesmittel finanzierten Geldleistungen der Arbeitsgemeinschaften (ARGEn), also das ALG II. Erst wenn Einkommen und Vermögen darüber hinaus zu berücksichtigen sind, mindert dies die Kosten der Unterkunft $(\$ 19$ SGB II). Aufstocker beziehen also sehr häufig nur geringes ALG II, bekommen jedoch die vollen Kosten der Unterkunft durch die Kommunen ersetzt.

Der deutsche Städtetag beklagte schon im Jahresfinanzbericht 2005, dass die Kommunen für die zusätzlichen Belastungen aus Hartz VI nicht in ausreichendem Maß Mittel zur Verfügung hätten. Die Summe der Zuweisungen durch die Länder sei nur um 2,3 Mrd. € gegenüber 2004 gestiegen, obwohl die Ausgaben für Soziales um 3,7 Mrd. € zugenommen hätten. Der Jahresfinanzbericht 2006 verweist auf die um $14 \%$ erhöhten Ausgaben für die Kosten der Unterkunft von Grundsicherungsbeziehern. Allein dadurch sind bei den Kommunen Ausgaben in Höhe von 13,8 Mrd. $€$ entstanden. Erneut blieb der Zuwachs bei den laufenden Zuweisungen (0,4 Mrd. €) hinter dem Anstieg der Sozialausgaben (1,9 Mrd. €) zurück. Die Länder profitierten offensichtlich von ihrer Mittlerposition. Sie konnten die Entlastung aus Bundesmitteln unvollständig weiterreichen, den Finanzausgleich zulasten der Kommunen modifizieren oder Zuweisungen für Investitionen kürzen (Pohl 2006, S. 59).

Diese durch die Einführung der Grundsicherung bei den Kommunen generierten zusätzlichen Haushaltsbelastungen werden auch deutlich, wenn man einen Blick auf die vierteljährliche Kassenstatistik wirft, in der die Ausgaben der Kommunen im Rahmen des SGB II ausgewiesen werden (Dietz et al.2006). Insgesamt stiegen die Ausgaben zwischen dem ersten Quartal 2005 und dem zweiten Quartal 2006 von 2 Mrd. € auf 3 Mrd. $€$. Da der Bund jedoch einen Teil dieser Ausgaben refinanziert, ist von einer Nettomehrbelastung von 1,5 bzw. 2 Mrd. $€$ im selben Zeitraum auszugehen. Vergleicht man die nun vorhandene finanzielle Belastung mit den Ausgaben für Sozialhilfe im Jahr 2004 kann man - trotz zusätzlicher Bundesmittel - eine kommunale Mehrbelastung von nahezu 500 Mio. $€$ im ersten Halbjahr 2006 feststellen.

Die kommunalen Ausgabensteigerungen bzw. die zugrunde liegende Finanzierungsstruktur führte zu erheblichen Konflikten zwischen Bund und Kommunen über eine Umverteilung der Lasten (BMAS 2006b; Deutscher Städtetag 2006b). Im Ergebnis einigte man sich auf eine Anhebung des Bundesanteils im Jahr 2007 auf 31,8\%. Das entspricht voraussichtlich zusätzlichen Bundesmitteln in Höhe von 4,3 Mrd. €. In den Folgejahren soll der Bundesanteil auf Basis einer gesetzlich verankerten Anpassungsklausel errechnet werden, die die Zahl der Grundsicherungsbezieher berücksichtigt (Henneke 2006). Insgesamt zeigen die dargestellten Entwicklungen, dass die Umschichtungen - entgegen der ursprünglichen Entlastungsversprechen der Bundesregierung bei der Einführung von Hartz IV - zusätzliche Lasten für die Kommunen hervorgebracht haben. Da diese Lasten aus dem System der Grundsicherung kommen, ist auch bei anziehender Konjunktur nur verzögert mit Besserung zu rechnen.

\section{Sparen auf Kosten der sozial Schwachen?}

Die Analyse der Verteilung der Kosten auf die Rechtskreise SGB III (Arbeitslosengeld I) und SGB II (Arbeitslosengeld II) macht deutlich, dass Mehrausgaben im SGB II Einsparungen im SGB III und bei den Maßnahmen der aktiven Arbeitsmarktpolitik gegenüberstehen. Insgesamt können wir eher von einer Kostensenkung ausgehen. Für Leistungen im Rahmen des Arbeitslosengeldes I (und Insolvenzgeld) wurden zwischen 2004 und 2005 2,3 Mrd. $€$ weniger ausgegeben. Hinzu kamen Einsparungen bei Maßnahmen der aktiven Arbeitsmarktpolitik in Höhe von 5,5 Mrd. €. Insgesamt konnten so die Gesamtausgaben der Bundesagentur für Arbeit für beide Rechtskreise um 1,4 Mrd. $€$ gesenkt werden (BA 2006). Im Jahr 2006 generierte die Bundesagentur aufgrund der Einsparungen im SGB III erneut Überschüsse, die sich auf 11,3 Mrd. $€$ belaufen werden (BA 2007). Besonders problematisch an dieser Sparpolitik ist, dass ihr - neben einem konjunkturbedingten Rückgang der Arbeitslosigkeit im Jahr 2006 - ein deutlicher Rückbau der Leistungen für aktive Arbeitsmarktpolitik, und zwar in beiden Rechtskreisen, zugrunde liegt.

\subsection{DIE SANIERUNGSPOLITIK IM RAHMEN DER ARBEITSLOSEN- VERSICHERUNG}

Der Einnahmeüberschuss bei der Arbeitslosenversicherung ist einerseits auf einen Anstieg der Beitragseinnahmen und auf den einmaligen Sondereffekt der Vorverlegung der Beitragszahlung der Arbeitgeber im Jahr 2006 zurückzuführen; andererseits auf die Mehreinnahmen infolge steigender Beschäftigung und Bruttolöhne. Ebenfalls dazu beigetragen hat die Trennung der Rechts- und Regelkreise - ein Aspekt, auf den wir im Folgenden genauer eingehen werden.

Der Evaluationsbericht zum organisatorischen Umbau der BA kritisiert eine „betriebswirtschaftliche und an versicherungslogischen Kalkülen orientierte Leistungspolitik der BA“, die insbesondere darauf abziele, „den Ressourceneinsatz so anzulegen, dass dessen Wirkung noch innerhalb der Bezugsdauer des ALG I eintritt“, 
richtigerweise als „Engführung der Zielausrichtung“ (Bender et al. 2006, S. 238f.). Auf diese Weise geraten die Probleme gesellschaftlicher Unterbeschäftigung und struktureller Arbeitslosigkeit aus dem Blickfeld. Die neue strategische Ausrichtung ist erkennbar eine „rationale Antwort der Organisation auf die Änderung ihrer Rahmenbedingungen“, die „die BA bislang auf die Herausforderung der institutionellen Trennung der Rechtskreise SGB III und SGB II und auf den Aussteuerungsbetrag bei Übertritt von Arbeitslosen nach SGB II gefunden hat $[\ldots]$. Sie steht jedoch in einem harten Spannungsverhältnis sowohl zum individuellen Unterstützungsbedarf ihrer Arbeitnehmerkunden als auch zu volkswirtschaftlichen, arbeitsmarktpolitischen und gesamtgesellschaftlichen Anforderungen" (ebd., S. 101 f.).

Denn die getrennten Rechtskreise und die damit verbundenen Finanzierungsstrukturen ermöglichen es der BA, ihren Erfolg im Kern an der Bilanz im Versicherungssystem zu messen, d.h. am Abbau der Arbeitslosigkeit im SGB III und an den erwirtschafteten Überschüssen, die in einen sinkenden Beitragssatz übersetzt werden können. Weitergehende soziale Konsequenzen von Arbeitslosigkeit, insbesondere verfestigte Langzeitarbeitslosigkeit und soziale Exklusion, werden der betriebswirtschaftlichen Versicherungslogik folgend in den Rechtskreis des SGB II geschoben. Gesamtgesellschaftliche Aufgaben von Qualifikation und Förderung Arbeitsloser, z. B. außerbetriebliche Ausbildungsplätze für Jugendliche ohne berufliche Perspektive, sind vernachlässigte Felder, die gesamtwirtschaftlich negativ zu Buche schlagen können.

Eines der bedeutsamsten Probleme, das aus dieser Vernachlässigung erwächst, ist der zunehmende Mangel an qualifizierten Fachkräften auf dem Arbeitsmarkt. Arbeitsmarktpolitik verschenkt hier eine Möglichkeit, positive Impulse für Wachstum und Beschäftigung zu geben. Gerade die Erfahrung früherer Konjunkturaufschwünge lehrt, dass mit anhaltender dynamischer Arbeitsnachfrage die Gefahr von Engpässen bei qualifizierten Arbeitskräften wächst. Hinzu kommen die demografischen Veränderungen der Erwerbsbevölkerung: Der Zustrom an jungen, gut ausgebildeten Arbeitskräften, die zukünftig aus den Ausbildungsstätten, Fach- und Hochschulen ins Erwerbsleben wechseln, wird abebben. Will man die Beschäfti- gungsfähigkeit einer älter werdenden Bevölkerung erhalten, werden in Zukunft höhere Investitionen in Weiterbildung notwendig sein.

\subsection{EINNAHMEN UND AUSGABEN DER GRUNDSICHERUNG}

Im Gegensatz zur Arbeitslosenversicherung sind die Ausgaben für erwerbsfähige Hilfebedürftige seit Hartz IV gestiegen. Die Bundesregierung veröffentlichte im Frühjahr 2006 einen Vergleich der Ausgaben vor und nach der Reform (Ausschussdrucksache 16(11)197): Danach lagen die Gesamtkosten 2005 um 15 \% über dem Vorjahresniveau. Der größte Anstieg war mit $303 \%$ bei den Kosten der Unterkunft zu verzeichnen. Die Zahlen für 2006 bestätigen diesen Trend. Die Gesamtausgaben ${ }^{3}$ stiegen um 3,8 Mrd. $€$ auf 42,1 Mrd. $€$; die Kosten der Unterkunft schlugen dabei mit 11,7 Mrd. $€$ (+1,3 Mrd. €) zu Buche.

Der Kostensteigerung liegt einerseits eine wachsende Zahl von Bedarfsgemeinschaften und Personen im Leistungsbezug, andererseits die Neuregelung der Kosten der Unterkunft (KdU) zugrunde. Die Zahl der Bedarfsgemeinschaften kann für die Zeit vor Inkrafttreten der Hartz-IV Reform nur geschätzt werden und wird für Anfang 2004 mit 2,86 Mio. angegeben (Rudolph 2004). Mit Inkrafttreten des SGB II im Januar 2005 waren 3,33 Mio. Bedarfsgemeinschaften mit 6,12 Mio. Personen im Leistungsbezug. Im Jahresmittel stiegen die Zahlen von 3,72 Mio. Bedarfgemeinschaften mit 6,76 Mio. Personen (2005) auf 3,95 Bedarfsgemeinschaften mit 7,29 Mio. Personen (2006) an. Hinter diesem Anstieg steht eine steigende Anzahl von Langzeitarbeitslosen (Arbeitsmarkteffekt), eine steigende Anzahl von Aufstockern sowie eine sinkende Dunkelziffer der Armut (Aust/ Müller-Schoell 2007).

Die beschriebene Umorientierung der BA auf Sparerfolge im SGB III übersetzte sich in zweierlei Hinsicht in ein wachsendes Problem der Grundsicherung: Die Schnittstelle zwischen Versicherungssystem (SGB III) und Fürsorgesystem (SGB II) ist über Aussteuerungsbetrag und Kundensegmentierung so gestaltet, dass Arbeitslose mit Vermittlungshemmnissen ohne kostenund zeitintensive Hilfen auf den Übergang ins Fürsorgesystem warten müssen. Dort steht faktisch ein eingeschränkter Katalog arbeitsmarktpolitischer Maßnahmen zur Verfügung (Aust et al. 2006). Der Rückgang der Anzahl der ALG I-Bezieher ist also auch Folge eines Creamingeffektes zulasten der Arbeitslosen, die schon von vornherein ein höheres Risiko haben, länger ohne Erwerbsarbeit zu bleiben, sodass insgesamt der Anteil der ALG II-Bezieher auf Kosten des Anteils der Bezieher des ALG I wachsen wird.

$\mathrm{Zu}$ dieser veränderten Strategie kommen gesetzliche Neuregelungen, die den „Nachschub für die ARGEn“ nicht abreißen lassen. Die Bezugsdauer des ALG I ist generell verknappt worden, insbesondere über kürzere Beitragszeiten für ältere Arbeitslose. Reduzierte bzw. keine Versicherungsansprüche arbeitsloser Personen im Arbeitslosenversicherungssystem führen zu einer zügigeren bzw. sofortigen Überweisung in die Grundsicherung. So erklärt sich, warum die Erfolgsmeldungen der BA mit Schwierigkeiten von ARGEn und Kommunen einhergehen. Vor diesem Hintergrund kommt die Evaluationsforschung zu dem Ergebnis, dass die konsequente Umsetzung einer betriebswirtschaftlichen Kostenreduzierung für die Versichertengemeinschaft dazu [...] führ[t], dass das Ziel einer frühzeitigen Intervention zur Vermeidung von Langzeitarbeitslosigkeit und damit zur Minimierung von Kosten für die Volkswirtschaft insgesamt sowie weitere [...] ökonomische und sozialpolitische Ziele zu weit aus dem Blickfeld der BA geraten" (BMAS 2006a, S. X) ist.

\subsection{SPAREFFEKTE ZULASTEN DER AKTIVEN ARBEITSMARKTPOLITIK}

Wie bereits oben erwähnt, lässt sich eine arbeitsmarktpolitische Sparpolitik feststellen, die sich insbesondere in einem deutlichen Rückbau der Leistungen für aktive Arbeitsmarktpolitik zeigt.

Der Eingliederungstitel und das Kapitel 3 im Haushalt der Bundesagentur weisen die Finanzmittel aus, die für Ermessens- und Pflichtleistungen der aktiven Arbeitsmarktpolitik im Rahmen des SGB III aufgewendet wurden (Tabelle 3). Dieser Aufstellung ist $\mathrm{zu}$ entnehmen, dass im Rechtskreis des SGB III die Ausgaben von 18,7 Mrd. $€(2004)$ über 13,56 Mrd. € (2005) auf 12,2 Mrd. € (2006) gesunken sind. Die größten Einsparungen gehen

\footnotetext{
3 Vorläufige BA-Daten ohne Optionskommunen
} (Adamy 2007, S. 9). 
Tabelle 3: Ausgaben für Arbeitsmarktpolitik im SGB III, 2004-2006 - in Mrd. €-

\begin{tabular}{|c|c|c|c|c|c|c|}
\hline & $\begin{array}{l}\text { Arbeits- } \\
\text { losengeld }\end{array}$ & $\begin{array}{l}\text { Kapitel-3- } \\
\text { Förderung }\end{array}$ & Davon: & $\begin{array}{l}\text { Eingliede- } \\
\text { rungstitel }\end{array}$ & Davon: & Davon: \\
\hline & & & $\begin{array}{l}\text { Existenz- } \\
\text { gründung }\end{array}$ & & Marktersatz & $\begin{array}{c}\text { Training und } \\
\text { FbW }\end{array}$ \\
\hline 2004 & 29,10 & 9,60 & 2,73 & 9,10 & 1,66 & 1,94 \\
\hline 2005 & 27,00 & 10,00 & 3,20 & 3,56 & 0,44 & 0,83 \\
\hline 2006 & 22,90 & 8,64 & 2,58 & 2,48 & 0,18 & 0,27 \\
\hline
\end{tabular}

Tabelle 4: Ausgaben für Arbeitsmarktpolitik im SGB II, 2005-2006 - in Mrd. € -

\begin{tabular}{|c|c|c|c|c|c|}
\hline & $\begin{array}{l}\text { ALG II und } \\
\text { Sozialgeld }\end{array}$ & $\begin{array}{c}\text { Eingliederungs- } \\
\text { titel }\end{array}$ & Davon: & Davon: & Davon: \\
\hline & & & $\begin{array}{l}\text { Beratung und } \\
\text { Qualifizierung }\end{array}$ & $\begin{array}{l}\text { Beschäftigungs- } \\
\text { begleitende } \\
\text { Zuschüsse }\end{array}$ & $\begin{array}{c}\text { Beschäftigung } \\
\text { schaffende } \\
\text { Maßnahmen } \\
\text { (Ein-€-Job, ABM) }\end{array}$ \\
\hline 2005 (Soll) & 14,6 & 5,18 & 1,27 & 0,91 & 2,17 \\
\hline 2005 (Ist) & 22,35 & 2,57 & 0,49 & 0,27 & 1,47 \\
\hline 2006 & 23,05 & 3,84 & 0,72 & 0,52 & 1,86 \\
\hline
\end{tabular}

dabei auf die Instrumente des Marktersatzes, also Arbeitsbeschaffungsmaßnahmen und Strukturanpassungsmaßnahmen, sowie die Förderung der beruflichen Weiterbildung zurück. Allein die Förderung von Existenzgründungen war zunächst von dieser Entwicklung ausgenommen. Aber auch hier greift mit dem neuen Gründungszuschuss 2006 der Sparkurs (Müller-Schoell 2006).

Auch im Rechtskreis des SGB II wurde der Eingliederungstitel im Jahr 2005 mit Ausgaben von 3,1 Mrd. $€$ im Vergleich zum Haushaltsplan nur zu $54 \%$ ausgeschöpft (Tabelle 4). Das bedeutet, dass die Maßnahmen zur Förderung der erwerbsfähigen Grundsicherungsbezieher weit unterhalb der geplanten Ausgaben von über 5,7 Mrd. € zurücklagen. Damit entgingen diesen Arbeitslosen beispielsweise Chancen zur Eingliederung in den ersten Arbeitsmarkt im Gegenwert von 711 Mio. $€$. Weitere 700 Mio. $€$ hätten für Beschäftigung schaffende Maßnahmen ausgegeben werden können. Um diese Beträge blieben die tatsächlichen Ausgaben nämlich hinter den geplanten Werten von 2,1 Mrd. € und 1,2 Mrd. € im Jahr 2005 zurück.

Auch wenn die Ausgaben für aktive Arbeitsmarktpolitik 2006 wieder anstiegen, kann dieser Trend höchstens als Konsolidierung auf niedrigerem Niveau interpretiert werden. Hinzu kommt die Tatsache, dass bei den Beschäftigung schaffenden Maßnahmen kaum noch Arbeitsbeschaf- fungsmaßnahmen (ABM) und Arbeitsgelegenheiten in der Entgeltvariante finanziert werden. An ihre Stelle sind die arbeitsmarktferneren Arbeitsgelegenheiten mit Mehraufwandsentschädigung (sogenannte Ein-Euro-Jobs) getreten.

\section{Regulativer Staat - Delegitimierter sozialer Auftrag}

Die fiskalischen Verschiebungen zwischen Bund, Versicherungssystem und Kommunen folgen keinem einheitlichen Muster. Tendenziell ist allerdings zu erkennen, dass der finanzielle Beitrag des Bundes zur Finanzierung der Arbeitslosigkeit rückläufig ist. Dies gilt für konkrete Geldmittel wie auch für die Defizithaftung. Die bundespolitische Entscheidung, sich weitgehend aus der konkreten finanziellen Verantwortung zurückzuziehen, stützt den beschriebenen Trend zur Pluralisierung und Sektoralisierung der einzelnen Felder der Sozialpolitik, was sowohl ihre finanzielle Verknüpfung als auch die politische Organisation der Interessenvermittlung angeht (Trampusch 2006). Diese regulative Wende steht einer koordinierten Bewältigung der Arbeitsmarktprobleme entgegen, wenn sie beispielsweise bei der BA eine Corporate Identity mit stark betriebswirtschaftlichen, auf das SGB III bezogenen Zielen befördert. Mit dieser Orientierung gehen dann eine Entkoppelung des SGB II und jeweils die Optimierung der eigenen Geschäftsergebnisse ohne Rücksicht auf gesamtgesellschaftliche Belange einher (Knuth 2006, S. 166). Ein wichtiger Faktor für den institutionellen Egoismus der BA ist ihre vormalige Krisensituation, die im Vermittlungsskandal und der anschließenden Reformdiskussion gipfelte. Seit Ende der 1990er Jahre stand die BA wegen ihrer relativen Erfolglosigkeit bei der Bekämpfung struktureller Unterbeschäftigung (weiterhin unterstelltes Ziel) bei gleichzeitigen Ausgaben von insgesamt 70-85 Mrd. $€$ in der Kritik (Streeck/Heinze 2003; HartzKommission 2002). Sie arbeitete ineffizient und zu teuer. Mit dem Ende der Bundeszuschüsse, also unter praktischem Sparzwang, bot es sich als Ausweis von Effizienz und für die Rückeroberung von gesellschaftlicher Akzeptanz an, Überschüsse zu erwirtschaften und Beiträge zu senken. In einem Zustand umfassenden politischen Misskredits erscheint die Vermeldung von scheinbaren Erfolgen, wie z. B. eines großen Überschusses und sinkender Beiträge, als eine rationale Strategie. Sinkende Lohnnebenkosten sind jedoch gesellschaftlich ein viel weniger drängendes Problem als wachsende Segmente der Prekarität oder der Ausschluss vom Arbeitsmarkt. Immerhin liegen die Lohnnebenkosten in Deutschland unter dem EU-Durchschnitt (Statistisches Bundesamt 2007).

Der enge Finanzrahmen generiert diesen institutionellen Egoismus nicht nur bei der BA, sondern auch bei den Kommunen. Auf kommunaler Ebene kann nicht mehr auf die Vorleistung aus einem Versicherungsverhältnis rekurriert werden, sodass der Kostendruck schnell in die Richtung umschlagen kann, den Leistungsbezug zu delegitimieren. Das Sparmotiv der BA richtet sich gegen mittelfristige Problemlösungen für arbeitsmarktferne Arbeitslose. Das Sparmotiv der Kommunen richtet sich gegen eine bedarfsgerechte Definition der Zielgruppe der Grundsicherung. Im Ergebnis werden langfristig all jene Bevölkerungsgruppen entlastet, die stabil im ersten Arbeitsmarkt integriert sind. Nachteile kumulieren bei den prekär Beschäftigten und den vom Arbeitsmarkt ausgeschlossenen Gruppen. 


\section{Gewinner und Verlierer}

Die Sparpolitik der BA trägt dazu bei, finanzielle Überschüsse zu erwirtschaften. Diese wiederum werden über die Absenkung der Sozialversicherungsbeiträge zur BA von $6,5 \%$ auf $4,2 \%$ an Arbeitnehmer (und Arbeitgeber) weitergegeben. Die teilweise Finanzierung der Beitragsentlastung aus der Mehrwertsteuererhöhung schlägt bei den Konsumausgaben von Arbeitnehmern, aber auch bei den Leistungsbeziehern zu Buche, wobei Letztere eben von der Beitragssenkung nicht profitieren.

Simulationsstudien können uns zeigen, dass sich für ca. $40 \%$ der alleinstehenden Langzeitarbeitslosen die finanzielle Situation verbessert hat. Ehemalige Arbeitslosenhilfebezieher, die bisher einen Anspruch auf ergänzende Sozialhilfeleistungen nicht realisiert haben, profitieren von der neuen Gesetzgebung (Bloß/Rudolph 2005, S. 4). Positiv ist darüber hinaus die Aufnahme erwerbsfähiger Empfänger der Grundsicherung in die gesetzliche Krankenversicherung zu bewerten. Weiterhin steht auf der Plusseite, dass die Versicherungsfreiheit für Empfänger von ALG II nach Vollendung des 55. Lebensjahres für in den Vorjahren privat versicherte Personen entfällt. Bei entsprechender Bedürftigkeit wird damit für ältere Erwerbsfähige ein Weg geöffnet, nach Jahren in der privaten Krankenversicherung in die Solidargemeinschaft der GKV zurückzukehren.

Für ca. 60 \% der ehemaligen Arbeitslosenhilfeempfänger hat sich allerdings die Einkommenssituation mit dem Systemwechsel verschlechtert. Betroffen davon sind insbesondere Personen, die in der Arbeitslosenhilfe relativ hohe Lohnersatzleistungen (53\% des letzten Nettolohns) bezogen. Sie werden nun auf die gültigen Regelsätze herabgestuft. Besonders problematisch ist dabei die Situation für ehemalige
Arbeitslosenhilfeempfänger mit Vollzeit beschäftigten Partnern, da sie aufgrund der verschärften Einkommensanrechnung den Anspruch auf Unterstützung häufig ganz verlieren (Becker/Hauser 2006, S. 80).

Der Übergang von Lohnersatzleistungen zu bedarfsgeprüften Anschlussleistungen bedeutet nicht nur einen finanziellen Einschnitt, sondern ist auch in biografischer Perspektive riskant: Denn wer einmal in den Bezug von Arbeitslosenhilfeleistungen geraten ist, hat geringere Chancen, wieder eine Versicherungsleistung zu erhalten (Bartelheimer et al. 2006, S. 16). Ansprüche können nur durch eine ausreichend lange Versicherungspflicht am ersten Arbeitsmarkt und nicht mehr durch die Teilnahme an Arbeitsmarktprogrammen erworben werden (Aust 2006).

Ein weiterer, aus der fiskalischen Logik des Systems resultierender Rückschritt ist die Absenkung der Beiträge zur Rentenversicherung (RV) bei den ALG II-Empfängern. Mit der Überweisung in das ALG II System erwerben Arbeitslose noch weniger Rentenansprüche als bislang; die Beiträge wurden nahezu halbiert. Führte ein Jahr ALG II-Bezug bislang zu einem Rentenplus von $4,28 €$ pro Monat, sind es ab dem Jahr 2007 nur noch 2,19€. Gleichzeitig wurde die Doppelversicherung aufgehoben. Dies betrifft vor allem sozialversicherungspflichtig Beschäftigte, die aufstockendes ALG II erhalten. Künftig sind Personen, die aus einem anderen Grund in der gesetzlichen Rentenversicherung pflichtversichert sind, nicht mehr über den SGB II-Träger versichert.

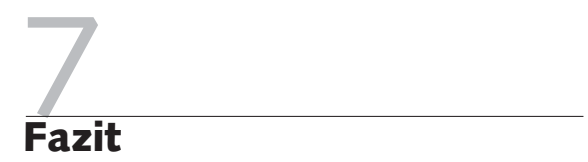

Die aktuell zu beobachtende konjunkturelle Erholung hat den Anstieg der Langzeitarbeitslosigkeit um sechs Prozentpunkte gegenüber dem Vorjahr nicht verhindern können (Adamy 2007). Vor diesem Hintergrund ist auch der moderate Rückbau der Mittel des Bundes, der sich an der Entwicklung der Gesamtausgaben im Sozialbudget erkennen lässt, problematisch. Unter dem Aspekt der Lastenverteilung zwischen bundesstaatlicher und kommunaler Ebene erweist sich die Hartz-Reform als Entlastung des Bundes zum Schaden der Kommunen. Im Verhältnis von Bund und Arbeitslosenversicherung kommt es zu einem weiteren Rückzug des Bundes aus der Verantwortung, was sowohl die eingesetzten Mittel als auch die Defizithaftung betrifft. Allerdings reagiert die BA auf diese Lage mit einer prononciert betriebswirtschaftlichen Handlungslogik, die einen Teil der Probleme an das Grundsicherungssystem weitergibt, wo letztlich die größten Kosten entstehen. Voraussetzung für diese strategische Ausrichtung ist die Trennung der Rechtskreise; verstärkend wirkt der Aussteuerungsbetrag, der Investitionen in arbeitsmarktferne Erwerbslose für die BA betriebswirtschaftlich irrational werden lässt. So kommt es in beiden Rechtskreisen zu einer fiskalisch motivierten Vernachlässigung von Instrumenten der aktiven Arbeitsmarktpolitik.

Die Hartz-Reform kann folglich als Rückzug des Bundes auf eine stärker regulative Funktion verstanden werden, die in der Folge bei den ausführenden Institutionen der Arbeitsmarktpolitik ein Interesse an reduziertem Maßnahmeneinsatz, reduzierten Leistungshöhen und einer Begrenzung der Zahl der Leistungsbezieher auslöst. Damit konzentrieren sich die Kostenvorteile bei Arbeitgebern und relativ gut integrierten Arbeitnehmergruppen, während die prekär ins Erwerbssystem integrierten und von ihm ausgeschlossenen Bevölkerungsgruppen Einbußen hinnehmen müssen. Ihr Leistungsbezug und ihre Förderung gerät direkt in den delegitimierenden Ruch der Fürsorge, die angesichts knapper Kassen immer unter Kürzungsvorbehalt steht. 
Adamy, W. (2007): Jahresbilanz 2006 - Perspektiven für 2007, in: Soziale Sicherheit 1, S. 5-14

Aust, J. (2006): Langzeitarbeitslos in Europa, in: WSI-Mitteilungen 8, S. 453-459

Aust, J./Bothfeld, S./Müller-Schoell, T./Schäfer, C. (2006): Zur Neudefinition des Verhältnisses von Arbeit und sozialer Sicherung. WSI-Thesen zur Workfare-Strategie in der Arbeitsmarktpolitik, http://www.monapoli.de/ cps/rde/xbcr/SID-3D0AB75D-CF3A5085/monapoli/WSI-Thesen_ Workfare_311106.pdf

Aust, J./Müller-Schoell, T. (2007): Vom Missbrauch einer Debatte, in: Rudolph, C./Niekant, R. (Hrsg.): Hartz IV - Zwischenbilanz und Perspektiven. Erfahrungsberichte, Analysen und Bewertung der bisherigen Umsetzung (im Erscheinen)

Bartelheimer, P./Baethge-Kinsky, V./Wagner, A. (2006): Zu den Auswirkungen von Hartz IV auf den Arbeitsmarkt - Fakten und Fragen, Interventionen 1, S. 14-31

Becker, I./Hauser, R. (2006): Verteilungseffekte der Hartz-IV-Reform, Berlin

Bender, G./Bieber, D./Hielscher, V./Marschall, J./Ochs, P./Vaut, S.

(2006): Organisatorischer Umbau der Bundesagentur für Arbeit. Evaluation der Maßnahmen zur Umsetzung der Vorschläge der Hartz-Kommission, Saarbrücken

Bloß, K./Rudolph, H. (2005): Simulationsrechnungen zum Arbeitslosengeld II. Verlierer, aber auch Gewinner, IAB Kurzbericht 17, Nürnberg Bundesministerium für Arbeit und Soziales (BMAS) (2006a): Die Wirksamkeit moderner Dienstleistungen am Arbeitsmarkt. Bericht 2006 des Bundesministeriums für Arbeit und Soziales zur Wirkung der Umsetzung der Vorschläge der Kommission Moderne Dienstleistungen am Arbeitsmarkt, Berlin

Bundesministerium für Arbeit und Soziales (BMAS) (2006b): Kommunalfinanzen

Bundesagentur für Arbeit (BA) (2006): Bericht über das vierte Quartal und das Geschäftsjahr 2005, Nürnberg

Bundesagentur für Arbeit (BA) (2007): Die Entwicklung des Arbeitsmarktes im Dezember und im Jahr 2006, Presse-Info 001 vom 3.1., Nürnberg Deutscher Städtetag (2006a): Aktuelle Finanzlage der Städte. Rückblick auf 2005 und Prognose für 2006, Berlin

Deutscher Städtetag (2006b): Haushaltsansätze des Bundes für Langzeitarbeitslosigkeit sind unrealistisch niedrig. Städte üben Kritik an Etatplanung für 2007, Pressemitteilung vom 4.7., Berlin

Deutscher Städtetag (2007): Aktuelle Finanzlage der Städte. Rückblick auf 2006 und Prognose für 2007, Berlin

Dietz, O./Müller, W./Schulze-Steikow, R. (2006): Öffentliche Finanzen im ersten Halbjahr 2006, in: Wirtschaft und Statistik 10, S. 1061-1067
Hartz-Kommission (2002): Moderne Dienstleistungen am Arbeitsmarkt. Vorschläge der Kommission zum Abbau der Arbeitslosigkeit und zur Umstrukturierung der Bundesanstalt für Arbeit, Berlin

Henneke, H.-G. (2006): Neuregelung der Finanzierungsverantwortung für die Leistungen für Unterkunft und Heizung nach § 46 Abs. 5 und 7 SGB II ab 2007, in: Der Landkreis 3, S. 128-131

Knuth, M. (2006): „Hartz IV“ - die unbegriffene Reform, in: Sozialer Fortschritt 7, S. 160-168

Künast, R./Andreae, K. (2007): Acht Reformschritte für die Handlungsfähigkeit der Kommunen. Kommunen stärken - Herausforderungen annehmen, in: Alternative Kommunalpolitik 1, S. 58-62

Müller-Schoell, T. (2006): Der Gründungszuschuss: Was bleibt von der Ich-AG nach der Zusammenlegung mit dem Überbrückungsgeld?, http://www.monapoli.de/cps/rde/xchg/SID-3D0AB75D-A2645DCC/ monapoli/hs.xsl/217.html

Naßmacher, H./Naßmacher, K.-H. (1999): Kommunalpolitik in Deutschland, Opladen

Paquet, R./van Stiphout, T. (2006): Hartz IV und die Auswirkungen auf die Krankenversicherung. Integration arbeitsmarktpolitischer Problemgruppen in die GKV, in: Sozialer Fortschritt 8, S. 192-195

Pohl, W. (2006): Gemeindefinanzbericht 2006. Vereinzelte Aufheiterungen, in: Alternative Kommunalpolitik 6, S. 58-61

Rudolph, H. (2004): Arbeitsmarkt-Reformen 2005. Aktualisierte Schätzungen zum Start von ALG II, IAB Kurzbericht 11, Nürnberg

Ruland, F. (2006): Rentenversicherung und Arbeitsmarkt, in: Deutsche Rentenversicherung 4-5, S. 225-238

Statistisches Bundesamt (2007): Arbeitskosten im europäischen Vergleich, Pressemitteilung vom 9.2., Wiesbaden

Streeck, W./Heinze, R. (2003): Optionen für den Einstieg in den Arbeitsmarkt oder: Ein Lehrstück für einen gescheiterten Politikwechsel, in: Vierteljahreshefte zur Wirtschaftsforschung 1, S. 25-35

Trampusch, C. (2003): Ein Bündnis für die nachhaltige Finanzierung der Sozialversicherungssysteme in der bundesdeutschen Arbeitsmarkt- und Rentenpolitik, MPIfG Discussion Paper 3, Köln

Trampusch, C. (2006): Status quo vadis? Die Pluralisierung und Liberalisierung der "Social-Politik" : Eine Herausforderung für die politikwissenschaftliche und soziologische Sozialpolitikforschung, in: Zeitschrift für Sozialreform 3, S. 299-323

Wahrendorf, V. (2006): Zur Angemessenheit von Wohnraum und Unterkunftskosten. Alte und neue Rechtsprechung zur "Wohnungsfrage" in SGB II, SGB XII und im BSHG, in: Soziale Sicherheit 4, S. 134-138 